\title{
Management of erosion of inflatable penile prosthesis reservoir into bladder. A different approach
}

\author{
Volkan Izol, Mutlu Deger, Bahattin Kizilgok, Ibrahim Atilla Aridogan, Mustafa Zuhtu Tansug \\ Department of Urology, Faculty of Medicine, University of Çukurova, Adana, Turkey.
}

\begin{abstract}
Summary We report a rare case of erosion of an inflatable penile prosthesis reservoir into the bladder that was managed with a different approach from the literature by preserving the existing reservoir. Inflatable penile implant was applied to a 54-year-old male patient who had undergone with a robot-assisted radical prostatectomy operation due to localized prostate cancer 2 years before. Two months after the operation, the patient referred to our clinic with predominant symptoms of lower urinary tract system associated with scrotal pain and swelling. The urinary system ultrasonography (USG) and the lower abdomen magnetic resonance imaging (MRI) demonstrated that the reservoir of the penile prosthesis was in the bladder. Cystoscopy confirmed that the reservoir was in the bladder. According to literature the reservoir was surgically removed from bladder. After bladder repair, the rectus muscles were repaired creating a space between the rectus muscle and the skin, where the reservoir was placed. After postoperative observation, the patient was discharged without any infection and regression of the lower urinary tract symptoms. No problem was referred by using the penile prosthesis when at 1-month and 3-month follow up and the patient was not uncomfortable in this regard. In conclusion no drawback occurred by using the old reservoir.
\end{abstract}

KEY WORDS: Penile prosthesis; Reservoir; Erosion; Bladder.

Submitted 31 December 2018; Accepted 26 January 2019

\section{INTRODUCTION}

Erectile dysfunction (ED) is defined as the inability to achieve and or maintain a penile erection sufficient for satisfactory sexual performance (1). Surgical implantation of penile prosthesis can be recommended as a third-line treatment in patients who have failed pharmacotherapy or require a permanent solution (2).

Complications of penile prostethesis include postoperative infection, bleeding, hematoma and device malfunction. In the literature, complications of reservoir of inflatable penile prosthesis are rarely reported, such as reservoir herniation, migration, erosion into the adjacent structure (e.g. bladder, bowel), ectopic reservoir location, hematoma, bowel obstruction and vascular compression with arterial or venous thrombosis (3).

We report a rare case of erosion of the reservoir of inflatable penile prosthesis into bladder. In the present case, the reservoir was not removed as in other cases in the literature and differently from published cases it was placed in an area under the skin above the rectus muscles. The relevance of this case report is the demonstration of a different approach for the treatment of this condition.

\section{Case report}

A 54-year-old male patient underwent a robot-assisted radical prostatectomy operation due to localized prostate cancer two years before our observation, Because of erectile dysfunction after operation, the patient received sildenafil therapy without benefit from the treatment, so inflatable penile implant was placed in another center. Two months after the operation, the patient referred to our clinic with predominant symptoms of lower urinary tract system associated with scrotal pain and swelling. On physical examination, the right testicle was normal at palpation with minimal hydrocele and penile prosthesis was detected. Left testicle was normal and minimal hydrocele was observed. Scrotal ultrasonography reported a bilateral complicated chronic hydrocele. Laboratory findings showed at urinalysis the presence of 1605 leukocytes, with positive leukocyte esterase $(++)$ and nitrite (+); blood count showed WBC 6900/ $\mathrm{mm}^{3}$; serum procalcitonin level was $0.09 \mathrm{n} / \mathrm{ml}$ and erythrocytes sedimentation rate (ESR) $59 \mathrm{~mm} / \mathrm{h}$; urine culture was negative. Meropenem $500 \mathrm{mg}$ ter in die treatment was started after consultation with Infectious Diseases and Clinical Microbiology clinic. In addition to antibiotic therapy, diclofenac sodium, scrotal elevation and cold application were also provided. Despite the treatment, due to the persistence of the patient's complaints, urinary system ultrasonography (USG) and lower abdomen magnetic resonance imaging (MRI) were performed and it was demonstrated that the reservoir of the penile prosthesis was in the bladder (Figure 1). Cystoscopy confirmed the presence of the reservoir in the bladder.

\section{Surgical procedure}

A Pfannenstiel incision was made to expose the bladder and perivesical space and the bladder anterior wall was released, to reach where the reservoir entered in the bladder. The bladder wall was opened to remove the reservoir and afterthat bladder repair was performed. After bladder repair, the rectus muscles were repaired and a space was created between the rectus muscle and the skin (Figure 2A). The reservoir was kept in saline 


\section{Figure 1.}

MR scan showing erosion of the reservoir into bladder.

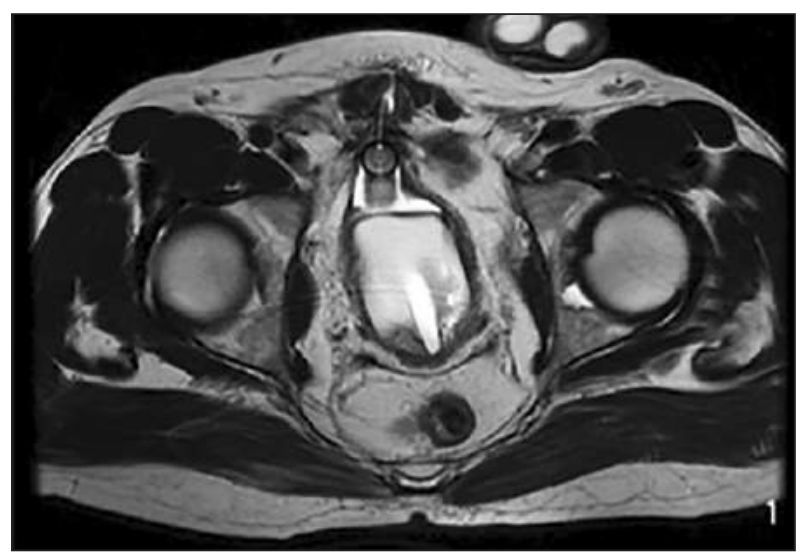

with diluted $180 \mathrm{mg}$ of gentamicin for 10 minutes. Then the reservoir was placed in the space formed (Figures 2B3). After the operation, the patient was treated with vancomycin and meropenem for 2 weeks. After postoperative observation, the patient was discharged without any infection and regression of symptoms of lower urinary tract. No problem in using the penile prosthesis was reported by the patient at 1-month and 3-month follow up. The patient was not uncomfortable in this regard because the patient's reservoir was not visible under the skin and computed tomography (CT) at 3 months did not show any infection status or abscess around the reservoir (Figure 4).

\section{Figure 2.}

A. Space was created between the rectus muscle and the skin. B: The reservoir was placed in the space formed.

A.

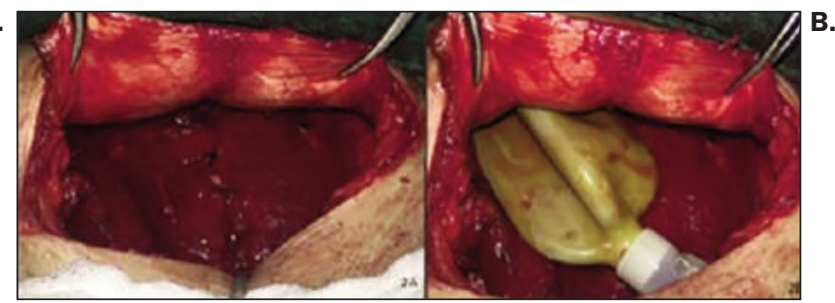

\section{Figure 3.}

The reservoir was placed in the space formed and semirigid penile erection was obtained.

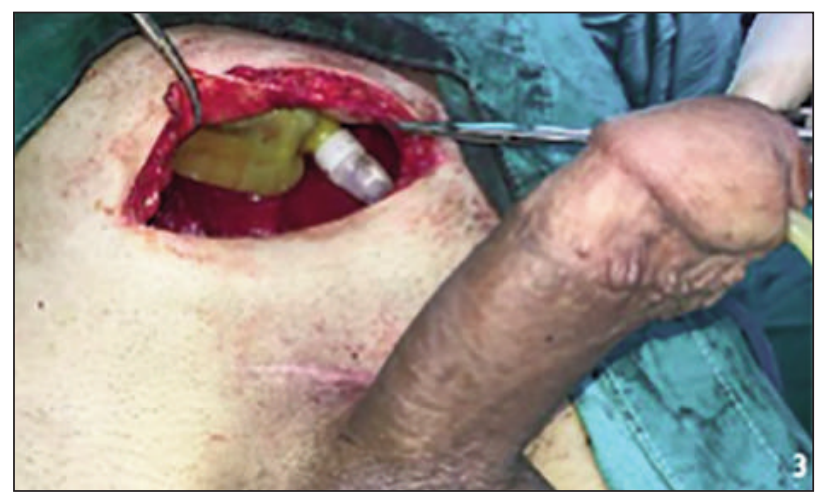

Figure 4.

CT scan at 3 months after operation showing the reservoir in the area formed without problem.

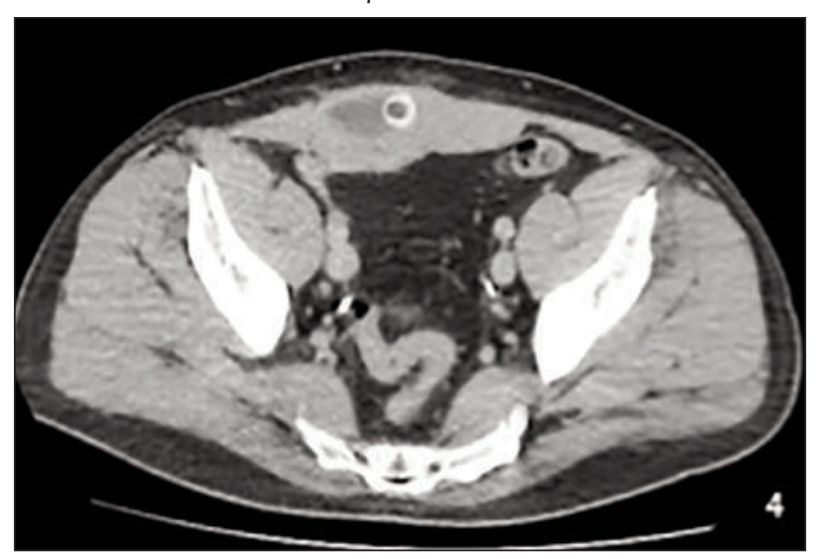

\section{Discussion}

Surgical implantation of penile prosthesis can be recommended as a third-line treatment in patients who have failed pharmacotherapy or require a permanent solution (2). There are two types of penile prosthesis used by urologists for erectile dysfunction: malleable (semi-rigid, non-inflatable) and inflatable. The first penile implant was introduced by Lash et al. in 1964 and Scott et al. introduced the first inflatable penile prosthesis (IPP) in 1973 (4). Inflatable penile prostheses (IPPs) consist of 3 pieces (two intracorporal cylinders, a scrotal pump and a fluid reservoir). These reservoirs may be placed in the space of Retzius, However, scarring after pelvic surgeries (such as radical prostatectomy, cystectomy, renal transplantation) destroys this space. In the literature, erosions of reservoir of inflatable penile prostheses into bladder have been reported. Possibly the first published case of IPP reservoir erosion into the bladder was described by Leach et al. in 1984. The patients were treated with complete explantation (5). In 1986, Fitch reported a case of reservoir erosion into bladder, but differently of Leach et al., he removed the old reservoir and placed a new reservoir with a successful outcome (6). In 1988, Dupont et al. reported an erosion of reservoir of IPP into the bladder after 4 years from initial implantation with formation of bladder calculi on the reservoir: a complete explantation was carried out (7). Park et al. reported a case of erosion of penile prosthesis reservoir into the bladder due to shortened tubing by multiple revision surgeries and suggested, as rescue surgery, the reposition of a new reservoir on the contralateral side to the erosion (8). In 2009, Kramer et al. report two cases of intravesical reservoir displacement presenting with gross hematuria in the recovery room following repair of a cylinder to pump tubing break of their inflatable penile prostheses (IPPs): they removed reservoirs and a new reservoir was placed in the contra-lateral space of Retzius (9). In 2012 Garber et al. report a case of erosion of a penile prosthesis reservoir into the bladder in a patient undergone four prior IPP surgeries and reported a literature review of intravesical erosions of penile implant reservoirs reporting that all penile implant reservoirs in the bladder mentioned in 
the literature were treated with complete explantation or placement of a new reservoir (10).

In 2013, Tran et al. present the case of a 75-year-old male with history of bladder cancer requiring radical cystoprostatectomy who had erosion of the inflatable penile prosthesis reservoir into the neobladder: the patient underwent removal of the IPP reservoir (11).

We report the case of the erosion of IPP reservoir into the bladder in a patient who had undergone robot assisted radical prostatectomy due to localized prostate cancer. Unlike other cases in the literature, we put the same reservoir in a space we had prepared after previous treatment of the infection without removal of the reservoir. The reservoir was placed in a space under the skin above the rectus muscle. This case is relevant because it offers a different new approach.

\section{Conclusions}

In conclusion the use of the old reservoir was safe. In addition, due to destruction of Retzius in pelvic surgery of some patients, such as those submitted to radical prostatectomy, we demonstrated that the reservoir could be placed in a safer area.

\section{REFERENCES}

1. NIH Consensus Development Panel of Impotence. JAMA. 1993; 270:83-90.

2. Hatzimouratidis K, Amar E, Eardley I, et al. Guidelines on male sexual dysfunction: erectile dysfunction and premature ejaculation. Eur Urol. 2010; 57:804-14.

3. Hartman RP, Kawashima A, Takahashi N, et al. Inflatable penile prosthesis (IPP): diagnosis of complications. Abdom Radiol (NY). 2016; 41:1187-96.

4. O'Rourke TKJr, Erbella A, Zhang Y, Wosnitzer MS. 'Prevention, identification and management of postoperative penile implant complications of infection, hematoma, and device malfunction. Transl Androl Urol. 2017; 6(Suppl 5):S832-S848.

5. Leach GE, Shapiro CE, Hadley R, Raz S. Erosion of inflatable penile prosthesis reservoir into bladder and bowel. J Urol. 1984; 131:11778.

6. Fitch WP $3^{\text {rd }}$, Roddy T. Erosion of inflatable penile prosthesis reservoir into bladder. J Urol. 1986; 136:1080.

7. Dupont MC, Hochman HI. Erosion of an inflatable penile prosthesis reservoir into the bladder, presenting as bladder calculi. J Urol. 1988; 139:367-8.

8. Park JK, Jang SW, Lee SW, Cui Y. Rare complication of multiple revision surgeries of penile prosthesis. J Sex Med. 2005; 2:735-6.

9. Kramer AC, Chason J, Kusakabe A. Report of two cases of bladder perforation caused by reservoir of inflatable penile prosthesis. $J$ Sex Med. 2009; 6:2064-2067.

10. Garber BB, Morris A. Intravesical penile implant reservoir: report, literature review, and strategiesfor prevention. Int J Impot Res. 2013; 25:41-4.

11. Tran CN, Boncher N, Montague DK, Angermeier KW. Erosion of inflatable penile prosthesis reservoir into neobladder. J Sex Med. 2013; 10: 2343-6.

\footnotetext{
Correspondence

Volkan Izol, MD

Mutlu Deger, MD, FEBU (Corresponding Author)

drmutludeger@gmail.com

Bahattin Kizilgok, MD

Ibrahim Atilla Aridogan, MD

Mustafa Zuhtu Tansug, MD

Department of Urology, Faculty of Medicine, University of Çukurova, Adana 01330 (Turkey)
} 